\title{
Application of Chinese ancient architecture elements in product design
}

\author{
YANG Mei ${ }^{1, a}$, YIN Huan ${ }^{2, b}$ * \\ ${ }^{1}$ Taiyuan University of Technology, China \\ ${ }^{2}$ Taiyuan University of Technology, China \\ a792152386@qq.com, b/158420468@qq.com
}

\begin{abstract}
Keywords: Chinese ancient architecture; product design; morphology; structure; organic combination.

Abstract. Research the method of blending Chinese ancient architecture into product design. From two sides of the overall features and characteristics of each part,summarized the artistic characteristics of Chinese ancient architecture. Enumerated examples of the comparison of morphology, structure and hue between Chinese ancient architecture and product design, and found the coherence point between them. Then summarized the method of applying Chinese ancient architecture to product design, and the method was put into practice to design a set of tableware. Organically blending Chinese ancient architecture into product design not only infuses flesh blood into the product morphology, but also makes it obtain new vigor.
\end{abstract}

\section{Introduction}

With thousands of years' accumulation, ancient Chinese traditional culture has deeply infiltrated into the ancient architectures. Consequently, the ancient architectures experienced vicissitudes have became the remaining Chinese treasure. We should not only protect, appreciate the treasure, but also inherit and develop it. In terms of product design, we should explore the available elements of the ancient architecture and apply its essence to modern product design. It is benefit to promote the cultural connotation of the products, meanwhile it also provides the source of inspiration for the product design. In addition, under the background of globalization, the design of a series of product with traditional Chinese charm is benefit to promote the national characteristics and board the arena of international design[1].

\section{The art characteristics of the ancient Chinese architecture}

The ancient Chinese architectures are the concrete image of the material culture created by the Chinese nation, which locate in the prosperous city or the streets, and even in the remote ancient village. The functions of the architectures are numerous, there are magnificent palaces, natural and decent dwellings, as well as the gorgeous classical garden[2]. However, all of them have architectural features, which is unique to China

The general characteristics. Owing to the special aesthetic feeling, the ancient architecture is enduring. In term of the overall outline, the architectural configuration has graceful curves. With thousands of years' vicissitudes, the architecture still stands erect, which can attribute to its wooden structure. The timber components connect with each other and add layer upon layer, thus the ancient architectures have distinct gradations, full appearance and artistic charm. In addition, the structure can also enhance the seismic behavior and safety performance of the houses[3].

The characteristics of each part .Main ridge: Located on the top of the roof, the main ridge parallels to the roof, which is the ridge ornament of the front and back slopes. In addition, it is an indispensable part of the ancient architecture. In different ages, the main ridges are not the same. In Han Dynasty, the both ends of the main ridge were slightly turnup, forming the state that two corners were upturned, which was especially popular in Tang and Song dynasties. In addition, it was straight after Ming Dynasty. However, even the straight main ridge also has upturned corners with shibi and Wang beast at the two ends[5]. These two kinds of main ridge are merely differ in arc curvature. 
Diagonal ridge for hip roof: The downward part that form the two ends of main ridge to the front and back slopes. Limited by the internal structure(the different variation range of the purline) of the roof. As the grade of the top roof is steep while the bottom is gentle, there will be an upward parabola between slop and diagonal ridge for hip roof[6]. The interaction between the Diagonal ridge for hip roof and the entirety can strengthen the roof contour. Therefore, the roof of ancient architectures have some functions, such as rain-proof and lighting.

Bracket set:Bracket set is the most outstanding invention of ancient Chinese architecture. Bracket set is formed by bucket block and arched short timber' s overlap on the chapiter. The roof' s power on the stringer can be passed to the bracket set, and then all the power will be transferred to the chapiter, which can eliminate the danger of beam breaking. That is the real reason for the phenomenon that" the wall collapsed but the house stands" .Decorative pattern:As one of the Chinese historical and cultural heritage, the decorative patterns exist in everywhere of the ancient architectures. Such as handrail, lintel, window, QT, etc.. There are many simple geometric figures as well as abstract organic patterns. In addition, people also skillfully combine the patterns to create many new luck meanings, which express people' s wishes and expectation, implicitly and vividly. The cracked ice are formed by simple geometric figures, and its shape is irregular. Looking from a distance, it looks like the melted snow. It is in stark contrast with the regular patterns, meanwhile it' s a natural and harmonious symbol that symbolizes the end of unhappy things and the possibility to realize the good wishes.

\section{The analysis of the ancient architecture elements' application in product design}

The referential method of the main ridge form. The design of the bench is shown in Fig.1, which is inspired by the main ridge of the ancient architecture. It only retain the most primitive and basic form of the bench, both ends of its surface are upturned slightly and forms a camber surface. The modification of the details are very smart, it not only avoid the rigidly connection between bench and the main ridge, but also optimize the function of the bench. In the past, when sitting on the edge of bench, it will be unstable. However, with the participation of the main ridge form, the users will be more safe sitting on the bench, and the tumble caused by instability can be avoided too. Excepting the reference of the main ridge form, the design also follows other features, such as the usage of wood and symmetry along the axis.

It shows that, the ancient architecture' s integration into the product design is not only the unnatural connection but also the organic combination of form and function[8], thus the result is that " $1+1>2$ $"$.

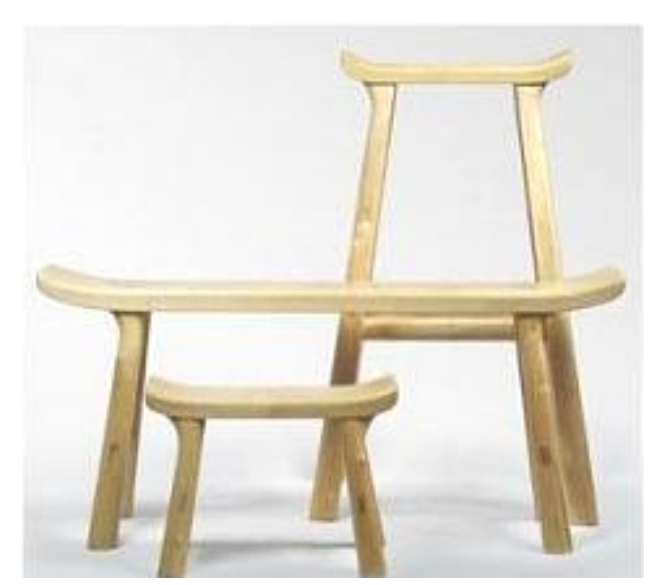

Fig.1 The design of bench 
The inheriting method of the diagonal ridge for hip roof' $\mathbf{s}$ form..In 2009, Brilliant Plumbing Group Co., Ltd. designed a series of faucet. Most of the faucets in the market are similar, however their designs can impress the people greatly, thus they are outstanding. This product is one of their design(refer Fig.2), the element of diagonal ridge for hip roof can be seen easily. In addition, the product also combines with the effect of eaves and walls, thus it seems that a part of architecture is moved to the washbasin without completely imitating. The design abstracts the radian of the diagonal ridge for hip roof and makes full use of it. The front of the product just like the eaves and walls connected in camber surface, which adds something soft to the overall shape. If the faucet is installed on the washbasin, it looks like a building. Once you turned the switch handle, the water will flow out from the water nozzle smoothly, which looks like that the drizzle slowly flows down the eave. Your hands will be washed by the rainwater.

The design references the form of diagonal ridge for hip roof and the charm of the stable ancient architecture, which greatly increase the ideal condition of the washbasin.

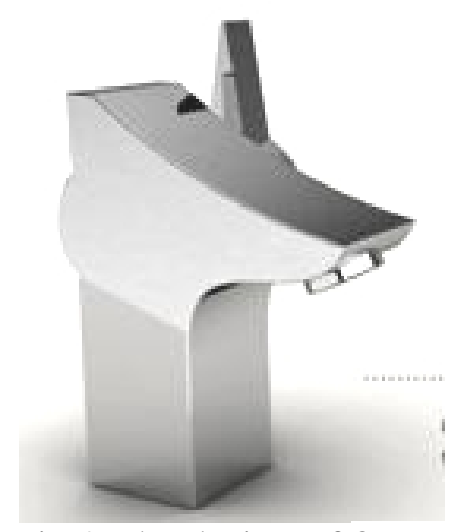

Fig. 2 The design of faucet

The extension methods of the bracket set.The application of bracket set elements can be divided into two categories: the first one is " form" (refer Fig.3), the design of the tea table uses the form of bracket set for reference, thus the tea table is full of rhythm; the other one is " spirit" , the most important part of the bracket set is the occlusion among the brackets, in addition, the brackets joints with each other in layers. The educational toy (refer Fig.4) adopts the structure of bracket set, the component connected with each other form an integrity. Although it seems quite different with the form of bracket set,it is highly consistent with its structure.

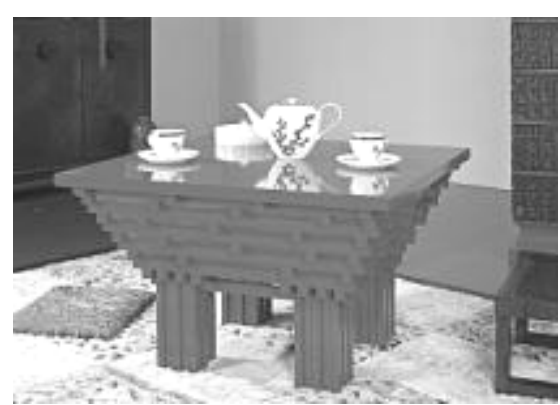

Fig. 3 The design of End table
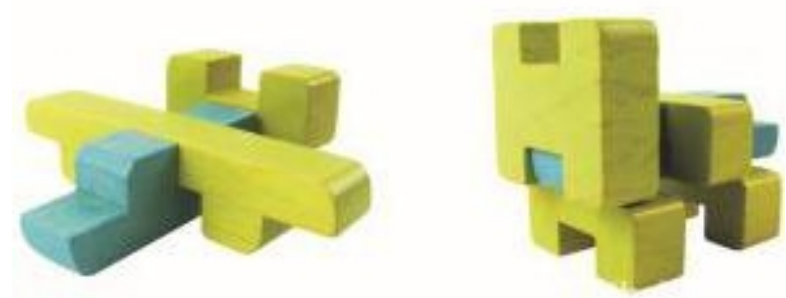

Fig.4 The design of educational toy

The application of the decorative patterns. The works shown in Fig. 5 is designed by the design master Jasper Hou. He adopts, in the ancient architecture, the elements of the windows used for dim beauty, and creates the modern clock that fulls of antique beauty. The traditional elements are timeless. Owing to the accumulation of the time, meanwhile it also creates unique aesthetic feeling, which deeply blend in our thought and blood. In addition, the clock also adopts cracked ice that conformed with golden section as well as the craft of" hollow out" . The pure black and white is the 
basic color of the clock, the striking red is the color matching for the needle and it also use the pure wooden material. All of them makes us feel refreshing. In this works, the function of the decorative patterns is self-evident. The combination of cracked ice and the craft of" hollow out" can not only show the time to the people, but also add certain art skill. It shows that the decorative patterns' application in the product design is not simple copy, we can make it more coordinated with the changes of color and skill.
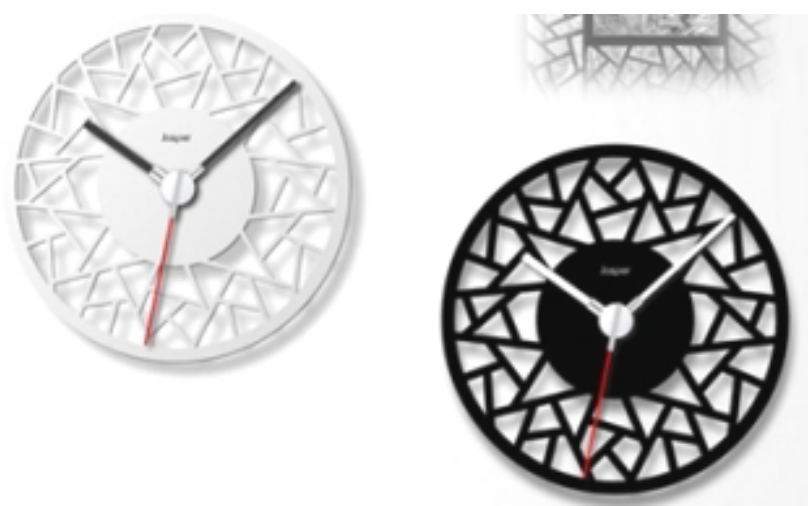

Fig.5 The design of clock

\section{Design practice}

In the design practice, the author chooses the elements of the ancient Chinese, such as architectures main ridge, diagonal ridge for hip roof, bracket set and decorative patterns, and works out the laws and methods using the previous analysis. Then through the draft plan, 3D modeling, render and other process, the author finally finishes the final effect, which is shown in Fig.10(the picture is painted by the author). The design follows the characteristics of the wooden material in the ancient architecture, and retains the feature of symmetry along the axis. Among which the form of plate uses the main ridge line for reference, while the curve of the saucer comes from the diagonal ridge for hip roof. In addition, the chopstick rest fully integrates with the features of bracket set, which is easy to assemble, disassemble and collect. It also adds some interest to the using process. The author also extracts the "longevity" from the window of ancient architecture and abstracts it, thus the word can be harmoniously integrated into the tableware designing .

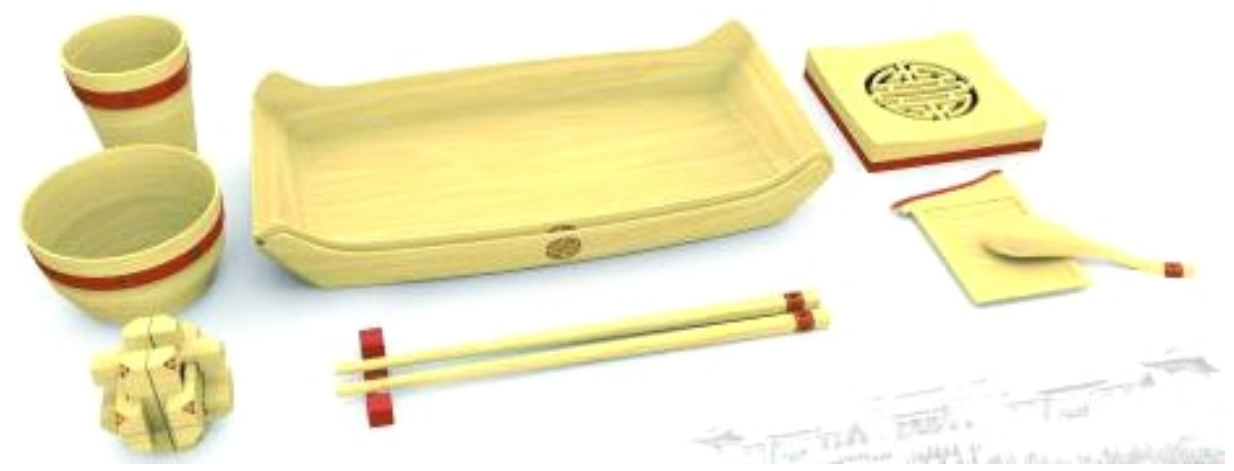

Fig. 6 The design of tableware

\section{Conclusion}

The inheritance and development of the ancient Chinese architecture elements cannot and should not be restricted into the visible materialized form, instead the details and connotation of the tradition are worth to be inherited[9]. The ancient architecture elements' application to the products should not 
simply copy and imitate the form but also combine with each other organically. The combination of form and function, the effective usage of the structure should be the integration of form and spirit, so as to rationally rereading the tradition when ensuring the continuation of the tradition. The design with ancient Chinese architecture elements can show the Chinese characteristics and national customs. This kind of design can makes the domestic users have intimacy, meanwhile it also conforms to the developing trend of internationalization[10]. As " The national things belong to the global" , the modern products with ancient Chinese architecture elements can help other countries to understand China as well as the ancient Chinese architecture through the different culture.

\section{References}

[1]Yimin WANG ,Yuanyan LIU . Influence of Form and Meaning of Intangible Cultural Heritage on Product[J]. Packaging Engineering,in Chinese2015(10):13-16.

[2]Yinong HUANG . Chinese Ancient Aesthetic Characteristics[J].Social Science of Hunan Normal University in Chinese, 1998(05):69-74.

[3]Hongtie ZHAO ,Fengliang ZHANG ,Jianyang XUE, etal. Research Review on Structural Performance of Ancient Timber Structure[J]Journal of Building Structures in Chinese, 2012(08):1-10.

[4]Dongtao .WANG Try to Talk About the Characteristics of aCchitectural in Ancient China[J]. Journal of Henan University ( Social Science) in Chinese,2005(04):131-133

[5]Hui XU ,Zhikui LIU . The Characteristic Analysis of Ridge Decoration of Ancient Chinese Architecture[J].Architectural Culture in Chinese, 2011(11):152-156.

[6] Ning GUO , Su LIU, Yunxing DU. Chinese Ancient Architecture Coof Causes the New Agent[J]. Studies in the History of Natural Sciences in Chinese, 2015(01):105-116.

[7]Lunhu XU . Code of Ancient Chinese Architecture(M). Beijing: Surveying and Mapping Press in Chinese,2010(01)

[8]Bihong LI. The Inovation of Contemporary Chinese Traditional Clture Elements in Art Expression[J]. Journal of Nanjing College of Art (Fine Arts \& Design) in Chinese,2015(03):171-177.

[9]Chunxiao QIN ,XIE Hengyuan. Application of Traditional Chinese Symbols in Product Design[J]. Packaging Engineering in Chinese,2008(05):131-132+153.

[10]Juexia ZHOU ,HU Lanzhen. Study on the Application of Traditional Chinese Culture in Product Design[J]. Packaging Engineering in Chinese,2013(12):84-86. 